BMJ Open

Diabetes

Research

\& Care

\title{
No consistent association between processes-of-care and health-related quality of life among patients with diabetes: a missing link?
}

\author{
Alejandra Casillas, Katia Iglesias, Aline Flatz, Bernard Burnand, \\ Isabelle Peytremann-Bridevaux
}

To cite: Casillas A, Iglesias $\mathrm{K}$, Flatz A, et al. No consistent association between processes-of-care and healthrelated quality of life among patients with diabetes: a missing link?. BMJ Open Diabetes Research and Care 2015;3:e000042. doi:10.1136/bmjdrc-2014000042

Received 17 June 2014 Revised 24 September 2014 Accepted 4 November 2014

CrossMark

Institute of Social and Preventive Medicine (IUMSP), Lausanne University Hospital, Lausanne, Vaud, Switzerland

Correspondence to Dr Alejandra Casillas; alejandra.casillas@hcuge.ch or Doctorale05@gmail.com

\section{ABSTRACT}

Purpose: Health-related quality of life ( $\mathrm{HRQOL}$ ) is considered a representative outcome in the evaluation of chronic disease management initiatives emphasizing patient-centered care. We evaluated the association between receipt of processes-of-care (PoC) for diabetes and HRQoL.

Methods: This cross-sectional study used selfreported data from non-institutionalized adults with diabetes in a Swiss canton. Outcomes were the physical/mental composites of the short form health survey 12 (SF-12) physical composite score, mental composite score (PCS, MCS) and the Audit of Diabetes-Dependent Quality of Life (ADDQoL). Main exposure variables were receipt of six PoC for diabetes in the past 12 months, and the Patient Assessment of Chronic IIIness Care (PACIC) score. We performed linear regressions to examine the association between PoC, PACIC and the three composites of HRQoL.

Results: Mean age of the 519 patients was 64.5 years (SD 11.3); $60 \%$ were male, $87 \%$ reported type 2 or undetermined diabetes and $48 \%$ had diabetes for over 10 years. Mean HRQoL scores were SF-12 PCS: 43.4 (SD 10.5), SF-12 MCS: 47.0 (SD 11.2) and ADDQoL: -1.6 (SD 1.6). In adjusted models including all six PoC simultaneously, receipt of influenza vaccine was associated with lower ADDQoL $(\beta=-0.4, p \leq 0.01)$ and foot examination was negatively associated with SF-12 PCS $(\beta=-1.8, p \leq 0.05)$. There was no association or trend towards a negative association when these PoC were reported as combined measures. PACIC score was associated only with the SF-12 MCS ( $\beta=1.6$, $p \leq 0.05$ ).

Conclusions: PoC for diabetes did not show a consistent association with HRQoL in a cross-sectional analysis. This may represent an effect lag time between time of process received and health-related quality of life. Further research is needed to study this complex phenomenon.

\section{BACKGROUND}

The number of people living with diabetes mellitus (DM) continues to rise, with an estimated 371 million individuals around the

\section{Key messages}

- In this study, processes of care for diabetes were not consistently associated with health-related quality of life when adjusting for patient and disease characteristics.

- There was similarly no association or trend towards a negative association when these processes of care were analyzed as combined measures.

- These findings prompt further diabetes research to focus on the effects of processes-of-care on health-related quality of life and other patientreported outcomes, from a patient-centered perspective, taking a longitudinal approach.

world who were affected by the illness in 2012. ${ }^{1}$ The consequences of diabetic illness, such as blindness and kidney disease, place a high toll on patients and the social system, not only in terms of functional life years lost, but also in terms of the morbidity that leads to disability and decreased quality of life. ${ }^{1-4}$ Given the significant impact of diabetes on patient lives and the responsibility of disease self-management on the individual with diabetes, health-related quality of life (HRQoL) is acknowledged as an important outcome to consider when developing and assessing interventions targeting these patients. ${ }^{5}$ Furthermore, novel chronic disease management initiatives emphasize a more "patientcentered' model, ${ }^{6}$ with increased attention to patient reported outcome measures (PROMs) such as HRQoL. ${ }^{7}$

However, although HRQoL is accepted as a diabetes-relevant outcome, having demonstrated positive associations with patients' daily life-functioning, disease management and prognosis, ${ }^{5-10}$ efforts for assessing the quality of diabetes care have primarily focused on the development and monitoring of process measures and clinical outcomes. For almost two decades, system initiatives in the USA and across the globe have poured 
resources into the implementation of specific process measures ${ }^{11-13}$ that improve relevant intermediate and ultimate end points such as glycated hemoglobin (HbA1C), and diabetic complications, comorbidities and overall prognosis, respectively. ${ }^{12}{ }^{14}{ }^{15}$ However, it is not yet known how these process measures affect PROMs in patients with diabetes, such as HRQoL.

Since process indicators are accepted as measures of high-quality diabetes care, ${ }^{16}$ we ask whether these established measures also ultimately translate into an improved patient-centered outcome. Therefore, the aim of this study was to examine whether receipt of highly utilized process(es)-of-care (PoC) measures was related to the HRQoL in patients with diabetes. We hypothesised that there would be a positive association between patient's receipt of PoC, as measured by individual and combined measures and HRQoL outcomes.

\section{METHODS}

Study design

We used data from a cross-sectional survey of patients with diabetes.

\section{Setting, population and recruitment}

In the fall of 2011 and summer of 2012, patients with diabetes were recruited by community-based pharmacies registered in the canton of Vaud, Switzerland, a large French-speaking canton (state) with over 720000 inhabitants. ${ }^{17}$ Patients were eligible if they came to the pharmacy with a prescription for oral hypoglycemics, insulin, glucometer and/or glucometer strips and had a diagnosis of diabetes for at least 12 months, were aged $\geq 18$ and non-institutionalized. Patients not residing in the canton of Vaud, not speaking or understanding French well enough, or those presenting with obvious cognitive impairment were excluded, as well as women with gestational diabetes. During this two-phase process, 85 pharmacies agreed to help recruit patients for the study and 519 eligible patients consented to the study, and completed and returned the questionnaire. Further details about recruitment and participation may be found in the published study about the original survey. ${ }^{18}$

\section{Measures}

Data were self-reported and collected using a paper mail-in survey that was given to patients during their visit to the pharmacy. It addressed the following themes: diabetes illness and care management, lifestyle, health status and sociodemographics.

\section{Dependent variables}

The main dependent variables of our study were two HRQoL measures, one generic and one disease-specific: the SF-12 and the Audit of Diabetes-Dependent Quality of Life (ADDQoL), respectively. We considered the mental composite score (MCS) and physical composite score (PCS) of the SF-12 rating, which each range from 0 (worst) to 100 (best). ${ }^{19}$ The 19-item ADDQoL instrument, ${ }^{20}{ }^{21}$ which is highly tailored to important issues of daily life for patients with diabetes, has been used among various types of populations, and is considered one of the few disease-specific tools with adequate psychometric properties. $^{22} 23$ The 19-item ADDQoL global score ranges continuously from -9 (worst) to 3 (best). Validation analyses for this project of the present French for Switzerland version showed a CFI (goodness-of-fit index) of 0.80 , and a Cronbach's $\alpha$ of at least 0.90 for all items.

\section{Exposure variables}

\section{PoC: individual measures}

Our main exposure variables were six individual PoC that are routinely used to monitor quality of diabetes care in practice and in research studies. ${ }^{12} 24$ The diabetes-specific patient-reported process measures were: HbA1C check (yes, $1 \times /$ year; yes $>1 \times$ /year; no; unknown), retinal eye examination by ophthalmologist (yes, $<1$ year ago; yes, 1-2 years ago; yes, >2 years ago; never; unknown), annual urine microalbuminuria screen (yes; no; unknown), annual foot examination by physician (yes; no; unknown), annual lipid test (yes; no; unknown) and annual influenza vaccination (yes; no; unknown). Owing to a skip question survey method, receipt of HbA1c check was only asked to those reporting knowing what HbAlc was (HbAlc-aware). However, multiple categories were created for the HbAlc PoC variable, to include the patients who did not know about HbAlc (HbA1c-unaware). The HbA1c variable categories were as follows: (1) HbAlc-aware and HbAlc was checked; (2) HbAlc-aware and HbAlc was not checked; (3) HbAlc-aware and do not know if HbAlc was checked; (4) HbAlc-unaware (not HbAlc-aware or do not know if HbAlc-aware). The reference category was 'HbAlc-aware and HbAlc was not checked.'

\section{PoC: combined measures}

We also used two combined measures for the PoC indicators. ${ }^{25}$ These combined measures were restricted to HbAlc-aware patients with no missing data, since these respondents were asked about HbAlc check and had answered all of the other PoC questions. This means that HbA1c-unaware patients and patients with missing and 'do not know' answers for any other PoC were not included in the following two combined measures:

1. Mean percentage of recommended care: the sum of received PoC (number of 'yes' responses per patient), divided by six possible processes.

2. Percentage of patients receiving all of recommended care: percentage of patients receiving six process measures (patients with all 'yes' responses), with reference to those not receiving all process measures (patients with 'no' for any of the six indicators).

\section{Patient Assessment of Chronic IIIness Care measure}

Our last exposure variable was the Patient Assessment of Chronic Illness Care (PACIC) global score (range from 
1-never to 5-always), which was used as an overall PoC measure, assessing to what extent current care is congruent with the Chronic Care Model. ${ }^{26}$ It does not incorporate the aforementioned PoC variables but, rather, bases its metrics on an evidence-based theoretical framework for chronic disease care.

\section{Covariates}

We considered other patient and clinical variables given their potential confounding with the association between PoC receipt and HRQoL. ${ }^{527-29}$ These variables included (1) demographics: age, gender, education (primary, secondary, tertiary), quartiles of monthly income level in CHF ( $\leq 3499,3500-5499,5500-9499$, $\geq 9500)$, living companion (lives with someone at home, yes/no), (2) medical history: alcohol consumption (history of alcohol problems, yes/no), smoking (currently smoking, yes/ no), body mass index (underweight/normal, overweight, obese), physical inactivity (no physical activity, yes/no), number of medical/psychiatric comorbidities and (3) diabetes characteristics and disease severity: diabetes type (type 2/undetermined, type 1), treatment (on insulin, yes/no), diabetes complications (presence of at least one of the following diabetes complications: cardiovascular disease, stroke, retinopathy, chronic kidney insufficiency, regular dialysis or kidney transplant, neuropathy, foot ulcer/wound, lower extremity amputation and severe hypoglycemia or hyperglycemia,) and duration of diabetes illness (10 years or more, yes/no). In order to not lose cases in fully adjusted models, missing and 'do not know' responses (if these two totaled over 10) were incorporated into a separate category for each variable.

\section{Statistical analysis}

First, we conducted descriptive analyses to describe the population, as well as HRQoL scores and PoC received. Next, we performed bivariate analyses to examine the association between each of the three HRQoL outcomes (SF12-MCS, SF12-PCS, ADDQoL) and each single PoC indicator. Using the ANOVA test, we assessed whether there were any differences in HRQoL mean scores between respondents for each PoC indicator, comparing report of receipt and no receipt of each PoC.

We then performed linear regressions of HRQoL outcomes and PoC. First, crude linear regression models for each of the three outcomes simultaneously included the six individual PoC (no covariates). All models were then adjusted for demographics, medical history and diabetes characteristics variables. Second, we used the two PoC combined measures and the PACIC to examine the association with the three HRQoL outcomes, in crude and adjusted models.

Finally, we performed a sensitivity analysis for the combined PoC measures to include all patient cases. This consisted of creating a combined measure to include patients with missing data as well as HbAlc-unaware patients who were not asked the HbAlc-receipt question (because they responded 'no' or 'do not know' to awareness about $\mathrm{HbA1c}$ ). In this sensitivity analysis (worst case scenario), the HbAlc-unaware patients were assumed as not having an HbAlc screen. Similarly, all patients with missing/'do not know' responses for each of the other indicators were counted as 'no' answers, thus giving a complete observation set of 519 patients for this combined measure sensitivity analysis. The sum of received PoC (number of 'yes' responses) was computed. We then used this combined measure to examine the association with the three HRQoL outcomes, in crude and adjusted models.

Checks for linearity and colinearity were performed in initial bivariate analyses. Residuals in each linear regression model were tested for normality and for homoscedasticity. We crosschecked all model results using transformed versions of the dependent variables, and these did not differ from the initial findings, nor did they provide a better residual distribution, which confirmed results of the original models used. To take into account the intragroup correlation of individuals within pharmacies, the clustered sandwich estimator was used to estimate the variance-covariance matrix (VCE). STATA V.12.0 was used for all analyses.

Written informed consent was obtained from all participants, and data were kept confidential.

\section{RESULTS}

Description of the study population is shown in table 1 . Among respondents, mean age was 64.5 years and almost $60 \%$ were men. The majority had a high school equivalent education or above $(81 \%)$ and lived with someone at home $(73 \%)$. While $17 \%$ were current smokers, $47 \%$ were classified as obese and nearly $30 \%$ were physically inactive. The average number of comorbidities per patient was 1.8. The majority of patients reported having type 2 diabetes $(67 \%), 12.7 \%$ reported type 1 diabetes; diabetes type diagnosis was unknown by $20 \%$ of respondents. Almost 50\% required insulin treatment and close to half self-reported at least one diabetes-related complication. The highest-reported diabetes complications were eye problems (with attention to the retina; 18\%), stroke or ischemic heart disease $(15 \%)$ and hypoglycemia or hyperglycemia $(11 \%)$. Finally, $48 \%$ of patients had had a diabetes diagnosis of greater than 10 years.

Table 2 presents the HRQoL outcomes and PoC results. Mean SF-12 MCS and PCS scores were 47.0 (SD 11.2) and 43.4 (SD 10.6), respectively, and mean ADDQoL score was -1.6 (SD 1.6). For individual process variables, about $98 \%$ of HbAlc-aware patients had had at least one HbAlc screen during the past 12 months. During that same time period, while screening for lipids was similarly high $(94.2 \%)$, around 60 $70 \%$ of the patients reported having received the other four individual process indicators, with the lowest corresponding to microalbuminuria screening (63.3\%). Combined PoC measures show that HbAlc-aware individuals received about $86 \%$ of the six PoC indicators 
Table 1 Characteristics of included patients with diabetes $(\mathrm{n}=519)$

\begin{tabular}{|c|c|}
\hline \multicolumn{2}{|l|}{ Demographics } \\
\hline Mean age $(n=519)$ & 64.5 (SD 11.3) \\
\hline Male $(n=519)$ & $59.7 \%$ \\
\hline \multicolumn{2}{|l|}{ Education $(n=504)$} \\
\hline Primary & $18.9 \%$ \\
\hline Secondary & $56.2 \%$ \\
\hline Tertiary & $25.0 \%$ \\
\hline \multicolumn{2}{|l|}{ Monthly income (CHF; $n=486)$} \\
\hline$\leq 3499$ & $21.2 \%$ \\
\hline $3500-5499$ & $26.5 \%$ \\
\hline 5500-9499 & $27.8 \%$ \\
\hline$\geq 9500$ & $17.3 \%$ \\
\hline 'do not know' & $7.2 \%$ \\
\hline Lives with someone $(n=516)$ & $73.2 \%$ \\
\hline \multicolumn{2}{|l|}{ Medical history } \\
\hline Current smoker $(n=509)$ & $17.3 \%$ \\
\hline History of alcohol problems $(n=505)$ & $5.9 \%$ \\
\hline \multicolumn{2}{|l|}{ BMI $(n=481)$} \\
\hline Underweight & $0.8 \%$ \\
\hline Normal & $17.1 \%$ \\
\hline Overweight & $35.3 \%$ \\
\hline Obese & $46.8 \%$ \\
\hline Mean number of comorbidities $(n=505)$ & 1.8 (SD 1.3) \\
\hline Physically inactive $(n=494)$ & $29.8 \%$ \\
\hline \multicolumn{2}{|l|}{ Diabetes characteristics } \\
\hline Diabetes type 2 or undetermined $(n=519)$ & $87.3 \%$ \\
\hline Diabetes requiring insulin $(n=516)$ & $48.8 \%$ \\
\hline Any diabetes complications* $(n=505)$ & $46.7 \%$ \\
\hline \multicolumn{2}{|l|}{ Diabetes duration ( $n=511$, years) } \\
\hline $1-5$ & $27.6 \%$ \\
\hline $6-10$ & $24.5 \%$ \\
\hline $11-15$ & $18.6 \%$ \\
\hline 16-20 & $11.9 \%$ \\
\hline over 20 & $17.4 \%$ \\
\hline \multicolumn{2}{|c|}{$\begin{array}{l}\text { *At least one diabetes complication: cardiovascular disease, } \\
\text { stroke, retinopathy, chronic kidney insufficiency, dialysis, } \\
\text { neuropathy, ulcer, amputation and severe hypoglycemia or } \\
\text { hyperglycemia. } \\
\text { BMI, body mass index. }\end{array}$} \\
\hline
\end{tabular}

considered, and about $40 \%$ of these patients received all six PoC. Results of the combined sensitivity analysis considering all 519 patients showed that at least $18.9 \%$ patients received six of the six processes-of-care; this is a result that tests a 'worst-case', where HbAlc-unaware for HbA1c, and missing and 'don't know' responses for any PoC receipt are considered as a 'no' answer.

The comparisons of mean HRQoL scores across categories of patients having reported $(+)$ or not $(-)$ a specific PoC are shown in table 3. Only a few comparisons were statistically significant. On the one hand, patients who received the influenza vaccine compared to those who did not, had lower ADDQoL scores $(-1.7$ vs -1.3 , respectively) and lower PCS scores (41.8 and 46.2, respectively), and those receiving the retinal examination reported worse ADDQoL score $(-1.7)$, compared to those who did not receive this examination $(-1.2)$. On the other hand, patients with HbAlc checks reported better ADDQoL than those who did not report any HbA1c check during the past 12 months. Of note, there were no significant differences in HRQoL outcomes between the HbAlc-aware and the HbAlcunaware groups (data not shown).

Crude and adjusted linear regressions of models including all individual PoC (table 4) suggest that while there were no associations between the receipt of single PoC and SF-12 MCS, receiving a foot examination by a physician was negatively associated with the SF-12 PCS score (ie, worse HRQoL: $\beta=-1.8$ ) in the fully adjusted model. Also, receipt of influenza vaccine was associated with lower ADDQoL in crude and in adjusted models $(\beta=-0.4)$.

Regarding combined measures of PoC (table 5), there was no association with HRQoL in the adjusted models. However, the sensitivity test ('worst case') did show that each additional $\mathrm{PoC}$ received was associated with a decrease in ADDQoL score $(\beta=-0.1)$.

Finally, PACIC was positively associated with HRQoL, with an increase in the SF-12 MCS score $(\beta=1.6)$ for each additional point on the PACIC global score (table 5).

\section{DISCUSSION}

The results of this study suggest that overall there were no consistently significant associations between patients' self-report of receipt of diabetic PoC and HRQoL. In fact, save for the PACIC score showing that care more congruent with the Chronic Care Model was positively associated with better SF-12 MCS score, and HbAlc being positively associated with better ADDQoL global score, there was either no association or a trend towards the negative. The findings were similar when we tested process indicators as combined measures.

These findings were somewhat unexpected since we hypothesized that receipt of PoC would lead, ultimately, to better clinical outcomes, and thus better HRQoL. Indeed, PoC have been shown to improve diabetic clinical markers such as HbAlc levels and cardiometabolic risk factors, ${ }^{30}$ thereby decreasing morbidity and mortality. ${ }^{14} 31 \quad 32$ Since less morbid patients report a better HRQoL, ${ }^{5} 3334$ we expected to see a positive association between PoC and HRQoL.

One possible reason for this discordance is that, in fact, receipt of PoC does not always translate to an observation of improved clinical outcomes for diabetes. In the third National Health and Nutrition Examination Survey (NHANES III), rates of health services use and diabetic complications screening and hyperglycemia, high-blood pressure and cholesterol treatment/therapy in patients with type 2 diabetes were high; however, despite the rise of these indicators, health status and outcomes did not reflect a significant improvement. ${ }^{35}$ Hypotheses for this finding included patient and healthcare system factors that did not allow for appropriately meeting patients' needs. Similarly, in the Translating Research Into Action 
Table 2 HRQoL scores and processes-of-care among included patients with diabetes $(n=519)$

\begin{tabular}{lll}
\hline HRQoL outcomes & $(\mathbf{n})$ & Mean (SD) \\
SF-12 MCS & 496 & $47.0(11.2)$ \\
SF-12 PCS & 498 & $43.4(10.6)$ \\
ADDQoL & 512 & $-1.6(1.6)$ \\
Individual process-of-care (at least one check in last 12 months) & & Percentage that received process \\
Hemoglobin A1c among 'aware' patients & 282 & 98.3 \\
Lipid & 513 & 94.2 \\
Urine microalbumin & 512 & 63.3 \\
Foot examination by physician & 510 & 66.5 \\
Retinal eye examination (in last 24 months) & 511 & 75.7 \\
Flu vaccination & 514 & 63.8 \\
Combined process-of-care measures & & \\
Mean percentage of recommended care* & 236 & 85.9 \\
Percentage of patients receiving all care & 236 & 41.5 \\
PACIC & & Mean (SD) \\
Global score & 503 & $2.8(0.95)$ \\
\hline
\end{tabular}

${ }^{*}$ Complete observations with no missing data (among HbA1c-aware patients only).

ADDQoL, Audit of Diabetes-Dependent Quality of Life; HRQoL, health-related quality of life; PACIC, Patient Assessment of Chronic IIIness Care; SF-12, short form-12

(TRIAD) study, though an average of one additional documented PoC for each patient in a group or plan was associated with significantly lower mean low-density lipoprotein cholesterol levels, this was not observed with HbA1c, systolic blood pressure or high-densitylipoprotein cholesterol levels. ${ }^{24}$ Finally, in a review of 24

Table 3 HRQoL outcome across individual processes-of-care, for those reporting it as having been performed (+) or not (-)

\begin{tabular}{|c|c|c|c|}
\hline & \multicolumn{2}{|c|}{ Health-related quality of life measures } & \multirow[b]{2}{*}{ p Value* } \\
\hline & $\begin{array}{l}\text { Mean (SD) } \\
\text { PoC not reported (-) }\end{array}$ & $\begin{array}{l}\text { Mean (SD) } \\
\text { PoC reported (+) }\end{array}$ & \\
\hline \multicolumn{4}{|l|}{ Lipids } \\
\hline SF-12 MCS & $48.4(9.1)$ & 46.9 (11.3) & 0.68 \\
\hline SF-12 PCS & $42.2(11.8)$ & $43.5(10.5)$ & 0.76 \\
\hline ADDQoL & $-1.5(1.4)$ & $-1.6(1.6)$ & 0.77 \\
\hline \multicolumn{4}{|c|}{ Urine microalbumin } \\
\hline SF-12 MCS & $46.6(11.4)$ & $47.4(11.1)$ & 0.54 \\
\hline SF-12 PCS & $41.6(9.8)$ & $44.0(10.6)$ & 0.12 \\
\hline ADDQoL & $-1.5(1.5)$ & $-1.6(1.6)$ & 0.48 \\
\hline \multicolumn{4}{|c|}{ Foot examination } \\
\hline SF-12 MCS & $46.7(11.0)$ & $47.2(11.2)$ & 0.66 \\
\hline SF-12 PCS & $44.1(9.0)$ & $43.1(11.2)$ & 0.59 \\
\hline ADDQoL & $-1.5(1.4)$ & $-1.6(1.6)$ & 0.72 \\
\hline \multicolumn{4}{|c|}{ Retinal eye examination } \\
\hline SF-12 MCS & $47.8(10.6)$ & 46.9 (11.3) & 0.39 \\
\hline SF-12 PCS & $43.8(11.0)$ & $43.2(10.5)$ & 0.38 \\
\hline ADDQoL & $-1.2(1.5)$ & $-1.7(1.6)$ & 0.01 \\
\hline \multicolumn{4}{|c|}{ Hemoglobin A1c } \\
\hline SF-12 MCS & $42.0(10.1)$ & $46.6(11.6)$ & 0.64 \\
\hline SF-12 PCS & 30.0 (14.9) & 43.7 (10.9) & 0.18 \\
\hline ADDQoL & $-2.8(0.98)$ & $-1.8(1.7)$ & 0.01 \\
\hline Flu vaccination & & & $\mathrm{p}$ value $\ddagger$ \\
\hline SF-12 MCS & 47.4 (10.9) & 46.8 (11.3) & 0.58 \\
\hline SF-12 PCS & $46.2(9.9)$ & $41.8(10.6)$ & 0.000 \\
\hline ADDQoL & $-1.3(1.3)$ & $-1.7(1.7)$ & 0.001 \\
\hline
\end{tabular}

${ }^{\star}$ F-test for ANOVA.

†Results displayed for $\mathrm{HbA1c}$-aware patients only.

$\mp$ Two sample t tests with equal variances.

ADDQoL, Audit of Diabetes-Dependent Quality of Life; ANOVA, analysis of variance; HbA1c, glycated hemoglobin; MCS, mental composite score; PCS, physical composite score; PoC, processes-of-care; SF-12, short form-12. 
Table 4 Linear regression models of HRQoL and individual processes-of-care in crude and adjusted models

\begin{tabular}{|c|c|c|c|c|c|c|}
\hline \multirow[b]{4}{*}{ Process indicators $†$} & \multicolumn{6}{|c|}{ HRQoL outcomes } \\
\hline & \multirow{2}{*}{\multicolumn{2}{|c|}{$\begin{array}{l}\text { MCS } \\
\beta=\end{array}$}} & \multirow{2}{*}{\multicolumn{2}{|c|}{$\begin{array}{l}\text { PCS } \\
\beta=\end{array}$}} & \multirow{2}{*}{\multicolumn{2}{|c|}{ ADDQoL }} \\
\hline & & & & & & \\
\hline & Crude & Adjusted & Crude & Adjusted & Crude & Adjusted \\
\hline Lipids & -2.6 & -3.4 & -0.05 & 2.1 & -0.005 & -0.03 \\
\hline Urine microalbumin & 1.2 & 0.95 & 1.8 & 0.5 & -0.1 & 0.05 \\
\hline Foot examination by physician & 1.1 & 0.9 & -1.5 & $-1.8^{*}$ & $-0.1^{*}$ & 0.2 \\
\hline Retinal eye examination (in past 24 months) & -0.7 & -0.2 & -0.5 & 1.1 & -0.3 & -0.2 \\
\hline Flu vaccination & -0.3 & -0.8 & $-4.2^{\alpha}$ & -1.4 & $-0.4^{\star *}$ & $-0.4^{\star *}$ \\
\hline \multicolumn{7}{|l|}{ Hemoglobin A1c } \\
\hline HbA1c-aware and HbA1c was checked & 4.8 & 1.4 & $14.9^{*}$ & 3.5 & 1.2 & 0.3 \\
\hline $\mathrm{HbA1c}$-aware and do not know if $\mathrm{HbA1c}$ was checked & 1.5 & -2.7 & $19.1^{*}$ & 4.6 & 2.2 & 0.8 \\
\hline $\begin{array}{l}\text { HbA1c-unaware (not aware or do not know if aware of } \\
\text { HbA1c) }\end{array}$ & 6.2 & 0.5 & $13.7^{*}$ & 3.8 & 1.6 & 0.4 \\
\hline Adjusted. $R^{2}$ for full model & 0.11 & & 0.39 & & 0.21 & \\
\hline
\end{tabular}

Crude model=all process indicator in one model, no other covariates.

Adjusted model=all process indicators+14 covariates (demographics, medical history and diabetes characteristics).

Covariates:

Demographics: Age, Male, Education levels, Income levels, Living companion.

Medical hx: hx of alcohol problems, smoker, BMI category, comorbidities, physical activity.

Diabetes: type 2 or undetermined, on insulin, DM complications, DM over 10 years.

${ }^{*} p \leq 0.05 ;{ }^{*} p \leq 0.01 ;{ }^{\alpha} p \leq 0.001$.

†Reference: no care received for individual process-of-care.

$\ddagger$ Reference: $\mathrm{HbA1c}$-aware and did not receive $\mathrm{HbA1c}$ screen/check.

$\mathrm{HbA1c}$, glycated hemoglobin; HRQoL, health-related quality of life.

studies looking at the association of diabetes quality-of-care with surrogate and clinical outcomes, high-quality studies specifically examining process indicators (number of tests or visits) showed mostly inconsistent or negative associations. ${ }^{36}$ Perhaps we could not find an improvement in HRQoL because the patients observed in this study did not clinically benefit from the PoC received at the time of the survey.

Another postulation is the confounding of severity of disease with more intense PoC, and thus low HRQoL.
We did try to control for this by adjusting for diabetesspecific characteristics and comorbidity; it is, however, still possible that we did not sufficiently account for all aspects of illness severity that are linked to lower HRQoL. In addition, increased medical treatment (such as increased doctor visits and medical testing) may lead to patient worry and inconvenience, and decreased patient HRQoL. Although we did not find studies documenting the effects of PoC on patient stress levels, the intensification of some medical treatment (such as the

Table 5 Crude and adjusted linear regression models of (1) HRQoL and combined processes-of-care, (2) HRQoL and PACIC, in crude and adjusted models

\begin{tabular}{|c|c|c|c|c|c|c|}
\hline & \multicolumn{6}{|c|}{ HRQoL outcomes } \\
\hline & \multicolumn{2}{|c|}{ MCS } & \multicolumn{2}{|l|}{ PCS } & \multicolumn{2}{|c|}{ ADDQoL } \\
\hline & Crude & Adjusted & Crude & Adjusted & Crude & Adjusted \\
\hline Mean percentage of recommended care $\dagger$ & 5.9 & 4.5 & -4.0 & -2.1 & -1.4 & -0.8 \\
\hline Adjusted $R^{2}$ for full model & 0.18 & & 0.35 & & 0.19 & \\
\hline Receiving all recommended care $†$ & 0.3 & 0.1 & -0.7 & 0.1 & -0.4 & -0.2 \\
\hline Adjusted $R^{2}$ for full model & 0.17 & & 0.35 & & 0.19 & \\
\hline Sensitivity analysisł & -0.1 & 0.2 & -0.4 & -0.3 & -0.2 & $-0.1^{*}$ \\
\hline Adjusted $R^{2}$ for full model & 0.12 & & 0.39 & & 0.21 & \\
\hline PACIC score & 0.96 & $1.6^{\star}$ & 0.5 & -0.6 & -0.1 & -0.1 \\
\hline Adjusted $R^{2}$ for full model & 0.13 & & 0.39 & & 0.20 & \\
\hline
\end{tabular}

Crude model=combined process-of-care measure, no other covariates.

Adjusted model=combined measure +14 covariates (demographics, medical history and diabetes characteristics).

Covariates:

Demographics: Age, Male, Education levels, Income levels, Living companion.

Medical hx: hx of alcohol problems, smoker, BMI category, co-morbidities, physical activity.

Diabetes: type 2 or undetermined, on insulin, DM complications, DM over 10 years.

${ }^{*} \mathrm{p} \leq 0.05$.

†Complete case: yes $=1 ;$ no $=0$; all other answers=missing.

fWorst case: yes=1; all other answers (HbA1c-unaware for $\mathrm{HbA1c}$; no, missing, do not know for all other PoC)=0. 
transition from oral hypoglycemics to insulin) has been linked to higher stress and worry and lower HRQoL among patients with type 2 diabetes, though this relationship was inconsistent. ${ }^{5}$

One more readily possible explanation is that the lack of association represents an effect lag time between time of process received and impact on HRQoL; cross-sectional data are not ideal to detect such an effect. Other studies have also described the need to measure quality of care and patient outcomes, over time, in order to most accurately reflect these relationships. ${ }^{10} 3738$ As such, the relationship between PoC and HRQoL might be further elucidated through a longitudinal examination, and thus should be a priority for future diabetic cohort studies.

Owing to this possible time-effect issue, one postulation was that receipt of PoC could be associated with a more immediate surrogate of patients' experiences, such as patient satisfaction. For example, the TRIAD study showed that the number of care processes documented was associated with patient satisfaction measures and self-rated quality of diabetes care. ${ }^{24}$ In post hoc analyses, we indeed observed a similar positive trend between PoC and 'very good/excellent' patient ratings on care satisfaction (vs 'bad/medium/good') and feeling 'very well/well informed' (vs 'very bad/bad/medium'), across individual and combined PoC measures, as well as the PACIC global score.

This study is unique in that we attempted to examine the specific association between PoC and HRQoL among a population-based sample of patients with diabetes, a topic that, to the best of our knowledge, has not yet been studied. We employed generic as well as diabetes-specific HRQoL outcomes, and a range of measurement for PoC (individual, combined, PACIC). However, our results need to be interpreted in light of the following limitations. First, since we used a crosssectional design, we cannot make conclusions about causal associations even though exposure to PoC was measured, retrospectively, for the previous year(s). Second, our sample size, calculated for getting appropriate precision around our primary outcomes, may have been too small to detect significant associations in secondary analysis. Another sample issue is that our combined analyses were limited to 236 of the 519 patients because of the skip question method for the question of HbA1c check receipt. In order to not eliminate the HbA1c-unaware patients from the combined measures, we constructed a third measure that was tested in the analysis where patients who responded 'no' or 'do not know' to knowing about HbAlc were assumed to not have received an HbA1c screen. A further point to consider is the relation between PoC measures and quality of life for patients with a specific complication (ie, how is quality of life affected in patients with retinopathy who receive an annual retinal examination compared to patients with diabetes who do not have retinopathy and receive annual retinal examinations?). Unfortunately, the sample size for many of these specific complication groups is much too small for further reliable analysis.
Third, the generalizability of these results is limited because the studied population may not be representative of the true population of patients with diabetes. We are nevertheless confident that our population-based sampling method, which utilized community pharmacies in the recruitment process, ensured the representativeness of patients with diabetes in the community (not just in clinic and hospital settings). Furthermore, characteristics of our patients with diabetes were similar to those reported from a population-based cohort study conducted in the same area. ${ }^{39}$ Last, we used self-reported data that may be prone to recall bias; however, supplementary analyses for this project demonstrated good correlation between patient-reported data and physician records for simple PoC, when data was supplied by the treating physician for a fraction of the cohort. ${ }^{40}$

In conclusion, further research should focus on the effects of PoC on patient-reported outcomes, particularly from a patient-centered perspective. Our study probes healthcare stakeholders to look beyond the presence of PoC procedures as the sole measurement of 'good care.' Additional studies, specifically with longitudinal data, are needed to examine the way in which PoC will translate into better health for patients with diabetes and, ultimately, a better quality of life. This includes looking into the way these PoC are conducted, how often they are delivered and what clinical cut-offs are being used. Although our study is a 'first look,' and more investigation is needed, we demonstrated that a list of achieved $\mathrm{PoC}$ is not a simple surrogate for gauging improvement on patient quality of life. Health systems and providers must ensure that diabetic care resources are allocated appropriately and organized effectively, so that patients with diabetes are able to thrive in life despite their illness.

Acknowledgements The authors would like to thank Emilie Zürcher, PhD for data management of overall study.

Contributors AC designed and conducted analyses, statistical interpretation and wrote the manuscript. KI provided statistical support, conducted ADDQoL validation analyses. AF gave feedback to the analysis and edited the manuscript. BB guided analyses, provided feedback and edited the manuscript. IP-B supervised all aspects of analyses, revised and helped to write the manuscript.

Funding This project was funded by the Department of Public Health of the canton of Vaud ('Programme cantonal Diabète'). IP-B was supported by a grant from the Swiss National Science Foundation (PROSPER no 32333B-123817 and no 32333B-139789) up to July 2013. Since August 2013, she has been supported by a grant from the Swiss School of Public Health (Assistant Professorship grant).

Competing interests None.

Ethics approval CHUV, Lausanne University Hospital.

Provenance and peer review Not commissioned; externally peer reviewed.

Data sharing statement No additional data are available.

Open Access This is an Open Access article distributed in accordance with the Creative Commons Attribution Non Commercial (CC BY-NC 4.0) license, which permits others to distribute, remix, adapt, build upon this work noncommercially, and license their derivative works on different terms, provided the original work is properly cited and the use is non-commercial. See: http:// creativecommons.org/licenses/by-nc/4.0/ 


\section{REFERENCES}

1. International Diabetes Federation. IDF Diabetes Atlas, 6th edn. Brussels, Belgium: International Diabetes Federation, 2013. http:// www.idf.org/diabetesatlas

2. Mokdad AH, Bowman BA, Ford ES, et al. The continuing epidemics of obesity and diabetes in the United States. JAMA 2001;286:1195-200.

3. Grandy S, Fox KM. Change in health status (EQ-5D) over 5 years among individuals with and without type 2 diabetes mellitus in the SHIELD longitudinal study. Health Qual Life Outcomes 2012;10:99.

4. Hoyert, DL. Xu, J. Center for Disease Control: Leading causes of death. National Vital Statistics Reports, Volume 61, Number 6. Oct 2012

5. Rubin RR, Peyrot M. Quality of Life and diabetes. Diabetes Metab Res Rev 1999;15:205-18.

6. AHRQ Health Care Innovations Exchange: innovations and tools to improve quality and reduce disparities, 2013. https://innovations. ahrq.gov/taxonomy-terms/endocrinologicmetabolic-care

7. Gibbons E, Fitzpatrick R. A structured review of patient-reported outcome measures for people with diabetes: an update 2009. University of Oxford, 2009. http://phi.uhce.ox.ac.uk/pdf/ PROMS_Oxford_KidneyReview_24112010.pdf

8. Collins $M M$. Quality of life and quality of care in patients with diabetes experiencing different models of care. Diabetes Care 2009;32:603-5.

9. McEwen LN, Kim C, Haan MN, et al. Are health-related quality-of-life and self-rated health associated with mortality? Insights from Translating Research Into Action for Diabetes (TRIAD). Prim Care Diabetes 2009;3:37-42.

10. Lau CY, Qureshi AK, Scott SG. Association between glycaemic control and quality of life in diabetes mellitus. J Postgrad Med 2004;50:189-93; discussion 94

11. de Beaufort CE, Reunanen A, Raleigh V, et al. European Union diabetes indicators: fact or fiction? Eur J Public Health 2003;13(3 Suppl):51-4.

12. Fleming BB, Greenfield S, Engelgau MM, et al. The Diabetes Quality Improvement Project: moving science into health policy to gain an edge on the diabetes epidemic. Diabetes Care 2001;24:1815-20.

13. McClain MR, Wennberg DE, Sherwin RW, et al. Trends in the diabetes quality improvement project measures in Maine from 1994 to 1999. Diabetes Care 2003;26:597-601.

14. De Berardis G, Pellegrini F, Franciosi M, et al. QuED (Quality of Care and Outcomes in Type 2 Diabetes) Study Group. Quality of diabetes care predicts the development of cardiovascular events: results of the QuED study. Nutr Metab Cardiovasc Dis 2008;18:57-65.

15. Nordwall M, Arnqvist HJ, Bojestig M, et al. Good glycemic control remains crucial in prevention of late diabetic complications-the Linkoping Diabetes Complications Study. Pediatr Diabetes 2009;10:168-76.

16. Nicolucci A, Greenfield S, Mattke S. Selecting indicators for the quality of diabetes care at the health systems level in OECD countries. Int J Qual Health Care 2006;18(Suppl 1):26-30.

17. Peytremann-Bridevaux I, Santschi V, Collet TH, et al. Community-based pharmacies: an opportunity to recruit patients? Int J Public Health 2013;58:319-22.

18. Zuercher E, Casillas A, Hagon-Traub I, et al. Baseline data of a population-based cohort of patients with diabetes in Switzerland (CoDiab-VD). Swiss Med Wkly 2014;144:w13951.

19. Beaton DE. Evaluating changes in health status: reliability and responsiveness of five generic health status measures in workers with musculoskeletal disorders. J Clin Epidemiol 1997;50:79-93.

20. Bradley C, Todd C, Gorton T, et al. The Development of an Individualized Questionnaire Measure of Perceived Impact of Diabetes on Quality of Life: The ADDQoL. Qual Life Res 1999;8:79-91.

21. Ostini R, Dower J, Donald M. The Audit of Diabetes-Dependent Quality of Life 19 (ADDQoL): feasibility, reliability and validity in apopulation-based sample of Australian adults. Qual Life Res 2012;21:1471-7.

22. El Achhab Y, Neijari C, Chikri M, et al. Disease-specific health-related quality of life instruments among adult diabetics: a systematic review. Diabetes Res Clin Pract 2008;80:171-84.

23. Speight J, Reaney MD, Barnard KD. Not all roads lead to Rome-a review of quality of life measurement in adults with diabetes. Diabet Med 2009;26:315-27.

24. Ackermann RT, Thompson TJ, Selby JV, et al. Is the number of documented diabetes process-of-care indicators associated with cardiometabolic risk factor levels, patient satisfaction, or self-rated quality of diabetes care? The Translating Research into Action for Diabetes (TRIAD) study. Diabetes Care 2006;29:2108-13.

25. Nolan T, Berwick DM. All-or-none measurement raises the bar on performance. JAMA 2006;295:1168-70.

26. Glasgow RE, Whitesides $\mathrm{H}$, Nelson $\mathrm{CC}$, et al. Use of the Patient Assessment of Chronic IIIness Care (PACIC) with diabetic patients: relationship to patient characteristics, receipt of care, and self-management. Diabetes Care 2005;28:2655-61.

27. Ko KD, Kim BH, Oh SI, et al. What are patient factors associated with the quality of diabetes care?: results from the Korean National Health and Nutrition Examination Survey. BMC Public Health 2012;12:689.

28. Lutfiyya MN, McCullough JE, Mitchell L, et al. Adequacy of diabetes care for older US rural adults: a cross-sectional population based study using 2009 BRFSS data. BMC Public Health 2011;11:940.

29. Wexler DJ, Grant RW, Wittenberg E, et al. Correlates of health-related quality of life in type 2 diabetes. Diabetologia 2006;49:1489-97.

30. Matthews DR. The natural history of diabetes-related complications: the UKPDS experience. United Kingdom Prospective Diabetes Study. Diabetes Obes Metab 1999;1(Suppl 2):S7-13

31. Saaddine JB, Cadwell B, Gregg EW, et al. Improvements in diabetes processes of care and intermediate outcomes: United States, 19882002. Ann Intern Med 2006;144:465-74.

32. Khanna A, Bush AL, Swint JM, et al. Hemoglobin A1c improvements and better diabetes-specific quality of life among participants completing diabetes self-management programs: a nested cohort study. Health Qual Life Outcomes 2012;10:48.

33. Garcia-Morales E, Lazaro-Martinez JL, Martinez-Hernandez D, et al. Impact of diabetic foot related complications on the health related quality of life (HRQol) of patients - a regional study in Spain. Int J Low Extrem Wounds 2011;10:6-11.

34. Maatouk I, Wild B, Wesche D, et al. Temporal predictors of health-related quality of life in elderly people with diabetes: results of a German cohort study. PLOS ONE 2012;7:e31088.

35. Harris MI. Health care and health status and outcomes for patients with type 2 diabetes. Diabetes Care 2000;23:754-8.

36. Sidorenkov G, Haaijer-Ruskamp FM, de Zeeuw D, et al. Review: relation between quality-of-care indicators for diabetes and patient outcomes: a systematic literature review. Med Care Res Rev 2011;68:263-89.

37. Miller DR, Pogach L. Longitudinal approaches to evaluate health care quality and outcomes: the Veterans Health Administration diabetes epidemiology cohorts. J Diabetes Sci Technol 2008;2:24-32.

38. Thompson $\mathrm{W}$, Wang $\mathrm{H}$, Xie $\mathrm{M}$, et al. Assessing quality of diabetes care by measuring longitudinal changes in hemoglobin A1c in the Veterans Health Administration. Health Serv Res 2005;40(6 Pt 1):1818-35

39. Firmann M, Mayor V, Vidal PM, et al. The CoLaus study: a population-based study to investigate the epidemiology and genetic determinants of cardiovascular risk factors and metabolic syndrome. BMC Cardiovasc Disord 2008;8:1-11.

40. Collet T-H, Taffé P, Bordet J, et al. Comparison of diabetes quality of care indicators as reported by patients and primary care physicians. Swiss Society of Internal Medicine, May 2013: IUMSP, 2013. 